\title{
BioéthiqueOnline
}

\section{Des enjeux éthiques liés à l'utilisation des données de recherches en collaboration internationale}

\section{Valéry Ridde}

Volume 6, 2017

URI : https://id.erudit.org/iderudit/1044621ar

DOI : https://doi.org/10.7202/1044621ar

Aller au sommaire du numéro

Éditeur(s)

BioéthiqueOnline

ISSN

1923-2799 (numérique)

Découvrir la revue

Citer cet article

Ridde, V. (2017). Des enjeux éthiques liés à l'utilisation des données de recherches en collaboration internationale. BioéthiqueOnline, 6 .

https://doi.org/10.7202/1044621ar
Résumé de l'article

Cette étude de cas en santé mondiale met au jour les enjeux éthiques associés à l'utilisation des données de recherche collectées dans le cadre d'une collaboration internationale ayant recours à des assistants de recherche. 


\title{
Des enjeux éthiques liés à l'utilisation des données de recherches en collaboration internationale
}

\author{
ÉTUde de CAs / CASE STUdY \\ Valéry Ridde ${ }^{1,2,3,4}$
}

Reçu/Received: 26 Oct $2017 \quad$ Publié/Published: 19 Dec 2017
Éditrices/Editors: Mariana Nunez \& Hazar Haidar

2017 Valéry Ridde, Creative Commons Attribution 4.0 International License

\section{Résumé}

Cette étude de cas en santé mondiale met au jour les enjeux éthiques associés à l'utilisation des données de recherche collectées dans le cadre d'une collaboration internationale ayant recours à des assistants de recherche.

\section{Mots clés}

santé mondiale, collaboration internationale, utilisation de données, thèse

\section{Abstract}

This global health case study uncovers the ethical issues associated with the use of research data collected in the context of international collaboration involving research assistants.

\section{Keywords}

global health, international collaboration, data use, thesis

\section{Affiliations des auteurs / Author Affiliations}

${ }^{1}$ Département de médecine sociale et préventive, École de santé publique de l'Université de Montréal (ESPUM), Montréal, Canada

${ }^{2}$ Institut de recherche en santé publique de l'Université de Montréal (IRSPUM), Montréal, Canada

${ }^{3}$ Centre Population et Développement (CEPED), Institut de Recherche pour le Développement (IRD), Université Paris

Descartes, Paris, France

${ }^{4}$ Équipe SAGE SUD, Institut national de la santé et de la recherche médicale (INSERM), Paris, France

\section{Correspondance / Correspondence}

Valéry Ridde, valery.ridde@umontreal.ca

\section{Conflit d'intérêts}

Aucun déclaré
Conflicts of Interest

None to declare

\section{Introduction}

Dans le domaine de la santé mondiale, de nombreuses organisations internationales ou non gouvernementales font appel à l'expertise des chercheurs et des professeurs d'universités du Sud et du Nord [1]. De surcroît, elles commissionnent souvent des recherches multi-sites, effectuées dans plusieurs pays, afin de comparer les résultats et augmenter le caractère transférable des conclusions. Ainsi, des collaborations internationales et le recours à des équipes importantes d'assistants de recherches voient-elles le jour dans plusieurs pays. Ces pratiques de recherches internationales impliquent des enjeux particuliers, notamment en ce qui concerne l'éthique, la collaboration et l'utilisation des données de recherche [2-4].

\section{Cas fictif inspiré de plusieurs histoires réelles}

Vous êtes professeur du CAMES ${ }^{1}$ dans un département de l'université du Gondwana. Vous êtes un spécialiste de la santé de la reproduction en Afrique. Depuis 20 ans, vous avez publié cinq livres et 76 articles scientifiques, dont trois dans une série spéciale The Lancet coordonnée par le Fonds des Nations Unies pour la population (FNUAP). Cette organisation internationale connaît vos qualités et

\footnotetext{
${ }^{1}$ Le Conseil Africain et Malgache pour l'Enseignement Supérieur est l'organisation régionale qui confère l'évolution des carrières des chercheurs et professeurs dans ses pays membres, cette évolution n'est pas régulée dans les universités mais par cette organisation.
} 
votre expertise. Elle décide donc de vous proposer une consultation pour coordonner une recherche multi-sites dans quatre pays du Sahel (Mali, Mauritanie, Niger et Tchad). L'objet de la recherche est de mieux comprendre pourquoi plus de $25 \%$ des femmes accouchent encore à la maison alors que ces quatre pays ont des politiques de gratuité des accouchements dans les formations sanitaires depuis cinq ans. Le FNUAP est intéressé par votre réseau africain afin que vous puissiez recruter des chercheurs experts sur le sujet dans ces pays et que vous en garantissiez la qualité des travaux. Ainsi, vous avez recruté deux professeurs et deux chercheurs des universités et centres de recherche de ces pays du Sahel.

La recherche commence en janvier 2016 avec un atelier de démarrage où vous décidez collectivement du protocole de recherche, des outils de collecte de données et des démarches à entreprendre dans chaque pays. Malheureusement, l'un de vos collègues professeurs est très occupé, il n'a pas pu venir à votre atelier car il s'était déjà engagé dans la réalisation d'une consultation de 10 jours pour une Organisation non gouvernementale (ONG) dans son pays. Mais vous le gardez dans l'équipe, il reste le responsable et a envoyé un de ses assistants pour l'atelier. Ce dernier est présent les quatre jours de l'atelier mais il n'a pas lu les documents préalablement, ne savait pas vraiment pourquoi il était présent et ne connaît pas bien le sujet. II a passé ses journées d'atelier devant son ordinateur à naviguer sur "Facebook », comme la collègue canadienne du FNUAP qui n'a pas semblé être plus intéressée par le sujet durant la rencontre.

La collecte des données démarre en mars et elle se déroule sans difficulté dans les quatre pays. Les chercheurs responsables ont tous recruté, comme prévu, trois assistants qui doivent, avec les chercheurs, réaliser les entrevues qualitatives dans deux districts sanitaires et 10 villages. II est prévu que les chercheurs responsables réalisent une dizaine d'entrevues avec les assistants pour que les premiers connaissent bien le terrain et s'assurent que les seconds comprennent bien le sujet et la démarche méthodologique qui reste complexe. Cependant, dans un des pays, vous apprenez par un de vos amis expatriés pour une ONG locale en agriculture, que le chercheur responsable n'est jamais venu sur le terrain. La totalité de la collecte des données a été réalisée par ses assistants, tous des étudiants inscrits à la maitrise en sociologie du développement rural dans son université du Nord du pays. En outre, ils se sont rendus dans les villages les plus proches, ils sont rentrés dormir tous les soirs dans la ville du district et n'ont visité que les formations sanitaires situées près de la route principale pour limiter leurs déplacements, car la route est de qualité exécrable en cette saison des pluies.

Lors de l'atelier final tenu en décembre 2016, qui vous permet d'effectuer une analyse comparative et collective avec les chercheurs, vous vous rendez compte, en effet, que le chercheur en question ne maitrise pas bien les résultats. II n'arrive pas à bien répondre aux questions que les collègues et le bailleur de fonds lui posent pour mieux comprendre certaines données empiriques. C'est pourtant essentiel pour vous permettre d'écrire le rapport comparatif. Ses réponses restent vagues, théoriques et n'apportent rien de nouveau sur le sujet « les femmes préfèrent accoucher chez elles, elles ne veulent pas accoucher avec du personnel masculin, si c'est gratuit les gens ne valorisent pas les soins ». Vous auriez pu dire la même chose sans faire cette recherche. Quelques mois plus tard, votre même ami de l'ONG vous apprend qu'il a assisté à la défense d'une thèse de doctorat en sociologie de la santé dont le titre est "Les accouchements à domicile en région rurale ». Intrigué, vous lui demandez une copie de cette thèse et découvrez qu'elle a été écrite par un des assistants de recherche du collègue que vous aviez recruté et que ce dernier, est même le directeur de thèse de l'étudiant.

\section{Questions}

1. Selon vous, quels sont les enjeux éthiques soulevés par cette étude de cas?

2. Quels défis posent-ils pour la conduite de la recherche?

3. Quelles actions auriez-vous prises si vous aviez été le responsable de ce programme de recherches? 


\section{Références}

1. Olivier C, Hunt MR, Ridde V. NGO-researcher partnerships in global health research: benefits, challenges, and approaches that promote success. Development in Practice. 2016;26(4):44455.

2. Godard B, Hunt M, Moube Z. Éthique de la recherche en santé mondiale : la relation NordSud, quel partenariat pour quelle justice sociale? Glob Health Promot. 2014;21(2):80-7.

3. Ridde V, Capelle F. La recherche en santé mondiale et les défis des partenariats Nord-Sud. Revue canadienne de santé publique. 2011;102(2):152-6.

4. Droz Y, Mayor A. Partenariats scientifiques avec l'Afrique : Réflexions critiques de Suisse et d'ailleurs. Paris: Karthala; 2009. 